\title{
Maternal obesity classes, preterm and post- term birth: a retrospective analysis of 479,864 births in England
}

\author{
Emma Slack, Kate E. Best, Judith Rankin and Nicola Heslehurst ${ }^{*}$ [D
}

\begin{abstract}
Background: Preterm ( $<37$ weeks gestation) and post-term birth ( $\geq 42$ weeks gestation) are associated with increased morbidity and mortality for mother and infant. Obesity (body mass index (BMI) $\geq 30 \mathrm{~kg} / \mathrm{m}^{2}$ ) is increasing in women of reproductive age. Maternal obesity has been associated with adverse pregnancy outcomes including preterm and post-term birth. However, the effect sizes vary according to the subgroups of both maternal BMI and gestational age considered. The aim of this retrospective analysis was to determine the association between maternal obesity classes and gestational age at delivery.

Methods: A secondary data analysis of 13 maternity units in England with information on 479,864 singleton live births between 1990 and 2007. BMl categories were: underweight $\left(<18.5 \mathrm{~kg} / \mathrm{m}^{2}\right)$, recommended weight $(18.5-24.9$ $\left.\mathrm{kg} / \mathrm{m}^{2}\right)$, overweight $\left(25.0-29.9 \mathrm{~kg} / \mathrm{m}^{2}\right)$ and obesity classes I $\left(30.0-34.9 \mathrm{~kg} / \mathrm{m}^{2}\right), \|\left(35.0-39.9 \mathrm{~kg} / \mathrm{m}^{2}\right)$, Illa $\left(40-49.9 \mathrm{~kg} / \mathrm{m}^{2}\right)$ and IIIb $\left(\geq 50 \mathrm{~kg} / \mathrm{m}^{2}\right)$. Gestational age at delivery categories were: Gestational age at delivery (weeks): extreme preterm (20-27), very preterm (28-31), moderately preterm (32-36), early term (37, 38), full term (39-40), late term (41) and post-term ( $\geq 42$ ). The adjusted odds of births in each gestational age category (compared to full-term birth), according to maternal BMl categories were estimated using multinomial logistic regression. Missing data were estimated using multiple imputation with chained equations.
\end{abstract}

Results: There was a J-shaped association between the absolute risk of extreme, very and moderate preterm birth and BMI category, with the greatest effect size for extreme preterm. The absolute risk of post-term birth increased monotonically as BMI category increased. The largest effect sizes were observed for class IIlb obesity and extreme preterm birth (adjusted OR 2.80, 95\% Cl 1.31-5.98).

Conclusion: Women with class IIIb obesity have the greatest risks for inadequate gestational age. Combining obesity classes does not accurately represent risks for many women as it overestimates the risk of all preterm and post-term categories for women with class I obesity, and underestimates the risk for women in all other obesity classes.

Keywords: Pregnancy, Obesity, Preterm, Post-term

\section{Background}

Preterm birth (<37 weeks gestation) complications were a leading cause of death in children under 5 years, accounting for 1 million mortalities globally in 2015 [1]. Preterm babies are at increased risk of complications such as cerebral palsy, autism and disability, with the risk increasing with decreasing gestational age at birth [2]. Post-term birth ( $\geq 42$ weeks gestation) is also

\footnotetext{
* Correspondence: nicola.heslehurst@ncl.ac.uk

Institute of Health \& Society, Newcastle University, Baddiley-Clark Building, Richardson Road, Newcastle upon Tyne NE2 4AX, UK
}

associated with an increased risk of stillbirth, neonatal and infant death [3-5], and an increased risk of maternal morbidity due to fetal macrosomia [6], caesarean section [7-9], haemorrhage [8] and thromboembolic disease [7].

Obesity (body mass index (BMI) $\geq 30 \mathrm{~kg} / \mathrm{m}^{2}$ ) in women of reproductive age is increasing. In the UK, $21.3 \%$ of women had an obese BMI in early pregnancy in 2016, and $28.4 \%$ had an overweight BMI $\left(25.0-29.9 \mathrm{~kg} / \mathrm{m}^{2}\right)$ [10]. In the USA, $31.8 \%$ of women aged $20-39$ had obesity in 2011-2012 [11]. The WHO divides obesity into sub-classes to reflect risk of co-morbidities: I (BMI

(c) The Author(s). 2019 Open Access This article is distributed under the terms of the Creative Commons Attribution 4.0 International License (http://creativecommons.org/licenses/by/4.0/), which permits unrestricted use, distribution, and reproduction in any medium, provided you give appropriate credit to the original author(s) and the source, provide a link to the Creative Commons license, and indicate if changes were made. The Creative Commons Public Domain Dedication waiver (http://creativecommons.org/publicdomain/zero/1.0/) applies to the data made available in this article, unless otherwise stated. 
$30.0-34.9 \mathrm{~kg} / \mathrm{m}^{2}$ ), II (BMI $35.0-39.9 \mathrm{~kg} / \mathrm{m}^{2}$ ) and III $\left(\mathrm{BMI} \geq 40 \mathrm{~kg} / \mathrm{m}^{2}\right)$. Often obesity classes are not used in pregnancy guidelines or clinical practice. However, there is evidence to support their use, for example the odds of women developing gestational diabetes increases from 3.01 (95\% CI $2.34-3.87$ ) for obesity class I, to 5.55 (95\% CI 4.27-7.21) for obesity class II/III [12]. Although class III obesity is the least prevalent obesity class, it is increasing at the most rapid rate over time, according to UK national data [13], and warrants further investigation. There is also an emerging interest in maternal extreme obesity (BMI $\geq 50 \mathrm{~kg} / \mathrm{m}^{2}$ ) due to the increase in risks among this population $[14,15]$. This presents an argument to further divide class III maternal obesity into IIIa $\left(40-49.9 \mathrm{~kg} / \mathrm{m}^{2}\right)$ and IIIb (BMI $\left.\geq 50 \mathrm{~kg} / \mathrm{m}^{2}\right)$.

Systematic reviews and meta-analyses show that maternal overweight and obesity are associated with preterm $[16,17]$ and post-term birth [18]. However, the effect sizes vary according to the subgroups of both maternal BMI and gestational age considered. For example, there is a lack of existing data on gestational age at birth and extreme obesity, and inconsistent definitions of gestational age categories used in published research [18] which do not reflect the sub-categorisations of term birth endorsed by the American College of Obstetricians and Gynecologists, the Society for Maternal-Fetal Medicine, and the Association of Women's Health, Obstetric and Neonatal Nurses [19].

In light of the inconsistent use of definitions of both gestational age and obesity classes, and the lack of data on extreme obesity, this study aimed to determine the associations between gestational age categories and maternal obesity classes.

\section{Methods}

This epidemiological study was a secondary analysis of an existing anonymised national dataset of routine maternity data NHS Trusts across England, UK [13]. The acquisition of the dataset has been described elsewhere $[13,20,21]$ and included data on live births from 37 maternity units (24 NHS Trusts) and $n=738,307$ births between 1989 and 2007. The dataset included booking information (i.e. first antenatal contact at approximately 12 weeks gestation) on measured maternal weight, measured maternal height, BMI calculated from measured height and weight, year, stage of pregnancy, maternal age, maternal ethnicity, maternal employment, parity, and Index of Multiple Deprivation (IMD) 2007 (a measure of area-level socioeconomic deprivation calculated from domains such as income, employment and health [22, 23], derived from mothers' residential postcode at booking). Within the dataset, 13 NHS Trusts provided gestational age at delivery data and therefore this subsample of the original dataset was used in this secondary analysis. Although this dataset contains information on live births, no information is provided on viability.

The outcome was gestational age at delivery which was coded into seven categories: extreme preterm (2027 weeks), very preterm (28-31 weeks), moderately preterm (32-36 weeks), early term (37-38 weeks), full term (39-40 weeks), late term ( 41 weeks) and post-term ( $\geq 42$ weeks). (Term births were categorised according to the American College of Obstetrics and Gynaecologists [24].) Below the limit of viability, 24 weeks [25], the chance of infant survival is very low, but not impossible [26]. Therefore, we included births $>20$ weeks gestational age. For extreme preterm birth, a sensitivity analysis was conducted, restricting to births $\geq 24$ weeks gestation to explore the decision to include birth below the usual limit of viability ( $>20$ weeks).

The main explanatory variable was maternal BMI, which was coded into seven categories: underweight $(<$ $\left.18.5 \mathrm{kgm}^{2}\right)$, recommended weight $\left(18.5-24.9 \mathrm{~kg} / \mathrm{m}^{2}\right)$, overweight $\left(25.0-29.9 \mathrm{~kg} / \mathrm{m}^{2}\right)$, obesity class I (BMI $30.0-$ $\left.34.9 \mathrm{~kg} / \mathrm{m}^{2}\right)$, class II $\left(\right.$ BMI $\left.35.0-39.9 \mathrm{~kg} / \mathrm{m}^{2}\right)$, class IIIa $\left(40-49.9 \mathrm{~kg} / \mathrm{m}^{2}\right)$ and class IIIb $\left(B M I \geq 50 \mathrm{~kg} / \mathrm{m}^{2}\right)$. Extreme obesity was defined as $B M I \geq 50 \mathrm{~kg} / \mathrm{m}^{2}$ to be consistent with previous publications [13, 14, 27]. BMI $<11 \mathrm{~kg} / \mathrm{m}^{2}$ and was recoded as missing as this is the lowest BMI for survival in women [28] and therefore it was assumed that any recorded BMI below this value was data entry error. BMI $>80 \mathrm{~kg} / \mathrm{m}^{2}$ was recoded as missing according to previously used limits [14, 29]. Further analyses were carried out collapsing the four maternal obesity categories into one obesity category (BMI $\geq 30.0 \mathrm{~kg} / \mathrm{m}^{2}$ ) to compare the findings with the analyses of maternal obesity classes I, II, IIIa and IIIb.

Additional socio-demographic explanatory variables were included in the adjusted analyses; variables chosen were hypothesised to be associated with both maternal obesity, and gestational age at delivery. These variables were: IMD (quintiles), ethnicity (White, South Asian, Black, Chinese/Other, Mixed ethnic group), employment (employed, not employed, home carer, higher education or education and/or age $<18$ ), maternal age at booking $(<20,20-24,25-29,30-34,35-39,40-44, \geq 45$ years $)$, gestational age at booking $(<13,13-25, \geq 26$ weeks) (please note that maternal age at booking and gestational age at booking were categorised due to non-linearity), parity (0 to 6) and region of England (East, London, North East, North West, South East, South West, West Midlands, Yorkshire and Humber) were analysed as categorical variables. Year of delivery was analysed as a continuous variable.

It was assumed the data were missing at random (where missingness can be explained by differences in observed data [30]). Therefore, multiple imputation using chained equations with 10 iterations was 
performed to impute missing data for BMI (17.9\% missing), IMD (1.4\%), ethnicity (15.0\%), employment (27.6\%), maternal age $(0.7 \%$ missing), parity $(1.7 \%)$ and gestational age at booking (0.4\% missing) [31]. All of these variables were included as predictors in the chained equations along with year of delivery and region (both complete), and gestational age at delivery. Gestational age at delivery was missing in $0.4 \%$ of pregnancies, these data were not imputed as this is not the recommended approach for the outcome variable [31]. Births with missing gestational age were excluded from the analyses.

Univariable multinomial logistic regression was performed with gestational age at delivery category as the outcome variable and BMI category as the explanatory variable. The model estimated the odds of each category of gestational age delivery compared to full term according to each category of BMI compared to the recommended category. Multivariable multinomial logistic regression was similarly performed adjusting for employment, ethnicity, maternal age, parity, gestational age at booking and year of delivery. Models were performed using both original and imputed data.

\section{Results}

The dataset included a total of 479,864 births between 1990 and 2007 of which 2954 (0.6\%) were extreme preterm, 3815 (0.8\%) were very preterm, 26,254 (5.5\%) were moderately preterm, $81,448(17.0 \%)$ were early term, 238,847 (49.8\%) were full term, 93,237 (19.4\%) were late term, 31,222 (6.5\%) were post-term and $2087(0.4 \%)$ had missing gestational age at delivery.

Following multiple imputation, $4.4 \%$ of women had an underweight BMI, 52.9\% had a recommended BMI, $27.3 \%$ had an overweight BMI, 10.7\% had class I obesity, $3.4 \%$ had class II obesity, $1.3 \%$ had class IIIa obesity, $0.1 \%$ had obese class IIIb obesity. Compared to the original dataset, in the imputed data there was a greater proportion of women in the categories of underweight (4.4\% vs $3.3 \%)$, overweight ( $27.3 \%$ vs $26.2 \%$ ) and obese class I (10.7\% vs $9.8 \%)$, and a lower proportion of women in the categories of recommended weight $(52.9 \%$ vs $55.6 \%)$, obese class II (3.4\% vs $3.6 \%)$ and obese class IIIa $(1.3 \%$ vs $1.5 \%)$. There was no difference for obese class IIIb $(0.1 \%$ for original data and imputed data)). Demographic information for IMD, employment, ethnicity, gestational age at booking, maternal age at delivery, parity, year of delivery and region are shown in Table 1 . There was a fairly even distribution of women across the IMD quintiles, the majority of women were White (84.9\%), employed (65.0\%), booked within the first trimester (59.4\%), aged $25-29$ (28.2\%) or 30.34 (26.7\%), had a parity of $0(36.6 \%)$ or $1(35.6 \%)$. Although data were available from 1990 to 2007 and for eight regions in England, the majority of included data were for births post 2002 (74.1\%) and women from the South East of England were represented more than other regions (39.3\%) (Table 1).

\section{Preterm birth}

There was a J-shaped association between the absolute risk of extreme preterm birth and maternal BMI category $(0.8,0.6,0.6,0.7,0.8,0.8$, and $1.4 \%$ for underweight, recommended weight, overweight and obese class: I, II, IIIa, IIIb respectively, Table 2). Compared with women in the recommended weight category, the AORs for extreme preterm birth were significantly increased for all obesity classes, with increasing effect size with increasing obesity class, most notably for obesity class IIIb (class I: 1.20 (95\% CI 1.03-1.40), class II 1.39 (95\% CI 1.13-1.71), class IIIa 1.52 (95\% CI 14.14-2.03), and class III3b 2.80 (95\% CI 1.31-5.98), Table 3). When combining all classes of maternal obesity into one obesity category, the AOR for extreme preterm birth was 1.33 (95\% CI 1.17-1.52, Table 4); higher than the AOR for class I obesity but lower than the AORs for classes II, IIIa and IIIb. The adjusted odds of extreme preterm birth were also significantly increased for maternal underweight and overweight categories (Table 3). The sensitivity analysis for extreme preterm birth when setting a higher gestational age limit to reflect the accepted limit of viability (24-27 weeks) identified an increased association with underweight, class II, IIIa and IIIb obesity compared with the AORs for extreme preterm birth when using the lower gestational age limit [20-27], and a decreased association for overweight and class I obesity (Table 3 ).

A J-shaped association was also observed between maternal BMI and very and moderate preterm birth (Table 2). The AORs for very preterm were significantly increased for maternal obesity classes I $(1.21,95 \% \mathrm{CI}$ $1.07-1.37)$ and II (1.35, 95\% CI 1.12-1.61), and for maternal underweight; however, there was no significant association with obesity classes IIIa/b or with maternal overweight (Table 3). The odds of moderate preterm birth were significantly increased for all obesity classes except IIIa; the greatest effect size was for obesity class IIIb: (1.07, 95\% CI 1.02-1.12; 1.13, 95\% CI 1.05-1.22; and $2.18,95 \%$ CI $1.58-2.99$ for classes I, II and IIIb respectively). There was also a significantly increased AOR for maternal underweight but not for maternal overweight (Table 3). When combining all maternal obesity categories, a similar pattern was observed for both very and moderate preterm birth with the AOR for overall obesity falling between the AORs for class I and II obesity (Table 4); therefore overestimating the risk for women with class I obesity and underestimating for the other obesity classes (although not all had significant associations for these two outcomes). 
Table 1 Study population socio-demographics, imputed and original data

\begin{tabular}{|c|c|c|c|c|c|}
\hline \multirow[t]{2}{*}{ Variable } & \multirow[t]{2}{*}{ Categories } & \multicolumn{2}{|c|}{ Original data } & \multicolumn{2}{|c|}{ Imputed data } \\
\hline & & $\mathbf{n}$ & $\%$ & n (estimated) & $\%$ \\
\hline \multirow{7}{*}{$\begin{array}{l}\text { Maternal } \\
\text { BMI }\end{array}$} & Underweight & 12,808 & 3.3 & 21,162 & 4.4 \\
\hline & Recommended & 218702 & 55.6 & 253,704 & 52.9 \\
\hline & Overweight & 103,062 & 26.2 & 131,099 & 27.3 \\
\hline & Obese class I & 38,731 & 9.8 & 51,154 & 10.7 \\
\hline & Obese class II & 13,139 & 3.6 & 16,171 & 3.4 \\
\hline & Obese class IIIa & 5,952 & 1.5 & 6,094 & 1.3 \\
\hline & Obese class IIIb & 508 & 0.1 & 528 & 0.1 \\
\hline \multirow{5}{*}{$\begin{array}{l}\text { IMD } \\
\text { quintile }\end{array}$} & 1 & 98,461 & 20.8 & 99,695 & 20.8 \\
\hline & 2 & 97,112 & 20.5 & 98,648 & 20.6 \\
\hline & 3 & 92,138 & 19.5 & 93,947 & 19.6 \\
\hline & 4 & 84,126 & 17.8 & 85,521 & 17.8 \\
\hline & 5 & 101,105 & 21.4 & 102,052 & 21.3 \\
\hline \multirow[t]{5}{*}{ Employment } & Employed & 223,109 & 64.2 & 312,074 & 65.0 \\
\hline & Higher education & 5,360 & 1.5 & 7,177 & 1.5 \\
\hline & Home carer & 75,028 & 21.6 & 104,332 & 21.7 \\
\hline & Unemployed & 35,306 & 10.2 & 46,469 & 9.7 \\
\hline & School/ Education & 8,811 & 2.5 & 9,813 & 2.0 \\
\hline \multirow[t]{5}{*}{ Ethnicity } & White & 343,316 & 84.2 & 406,141 & 84.6 \\
\hline & South Asian & 37,685 & 9.2 & 43,206 & 9.0 \\
\hline & Black & 13,690 & 3.4 & 15,316 & 3.2 \\
\hline & Chinese/ Other & 8,903 & 2.2 & 10,269 & 2.1 \\
\hline & Mixed & 4,253 & 1.0 & 4,932 & 1.0 \\
\hline \multirow{3}{*}{$\begin{array}{l}\text { Gestational } \\
\text { age at booking }\end{array}$} & 0-12 weeks & 276,215 & 60.1 & 285,125 & 59.4 \\
\hline & $13-25$ weeks & 137,074 & 29.8 & 147,398 & 30.7 \\
\hline & 26 weeks + & 46,150 & 10.0 & 47,341 & 9.9 \\
\hline \multirow[t]{7}{*}{ Maternal age } & $<20$ & 50,598 & 10.6 & 50,831 & 10.6 \\
\hline & $20-24$ & 99,486 & 20.9 & 100,055 & 20.9 \\
\hline & $25-29$ & 134,462 & 28.2 & 135,458 & 28.2 \\
\hline & $30-34$ & 127,462 & 26.7 & 128,337 & 26.7 \\
\hline & $35-39$ & 56,108 & 11.8 & 56,503 & 11.8 \\
\hline & $40-44$ & 8,276 & 1.7 & 8,365 & 1.7 \\
\hline & $45+$ & 306 & 0.1 & 315 & 0.1 \\
\hline \multirow[t]{5}{*}{ Parity } & 0 & 173,186 & 36.7 & 175,766 & 36.6 \\
\hline & 1 & 168,175 & 35.7 & 170,828 & 35.6 \\
\hline & 2 & 78,798 & 16.7 & 80,783 & 16.8 \\
\hline & 3 & 30,929 & 6.6 & 31,738 & 6.6 \\
\hline & $4+$ & 20,530 & 4.4 & 20,749 & 4.3 \\
\hline \multirow{3}{*}{$\begin{array}{l}\text { Year of } \\
\text { delivery }\end{array}$} & 1990-1995 & 38,103 & 8.0 & $\mathrm{n} / \mathrm{a}$ & \\
\hline & 1996-2001 & 86,092 & 17.9 & $\mathrm{n} / \mathrm{a}$ & \\
\hline & $2002-2007$ & 355,669 & 74.1 & $\mathrm{n} / \mathrm{a}$ & \\
\hline \multirow[t]{8}{*}{ Region } & East England & 10,541 & 2.2 & $\mathrm{n} / \mathrm{a}$ & \\
\hline & London & 68,225 & 14.2 & $\mathrm{n} / \mathrm{a}$ & \\
\hline & North East & 31,949 & 6.7 & $\mathrm{n} / \mathrm{a}$ & \\
\hline & North West & 54,488 & 11.4 & $\mathrm{n} / \mathrm{a}$ & \\
\hline & South East & 188,692 & 39.3 & $\mathrm{n} / \mathrm{a}$ & \\
\hline & South West & 78,282 & 16.3 & $\mathrm{n} / \mathrm{a}$ & \\
\hline & West Midlands & 7,696 & 1.6 & $\mathrm{n} / \mathrm{a}$ & \\
\hline & Yorkshire \& Humber & 39,991 & 8.3 & $\mathrm{n} / \mathrm{a}$ & \\
\hline
\end{tabular}

Footnote: Year of delivery and region were complete and therefore multiple imputation was not required. Abbreviations: BMI Body mass index, IMD Index of multiple deprivation, $n / a$ Not applicable 
Table 2 Estimated absolute risk of gestational age at delivery categories according to BMI category using original (non-imputed) and imputed data

\begin{tabular}{|c|c|c|c|c|c|c|c|c|c|}
\hline BMI category & $\begin{array}{l}\text { Extreme Preterm } \\
\text { (20-27 weeks), } \\
\mathrm{N}(\%)\end{array}$ & $\begin{array}{l}\text { Extreme Preterm } \\
(24-27 \text { weeks), } \\
n(\%)\end{array}$ & $\begin{array}{l}\text { Very } \\
\text { Preterm, } \\
\mathrm{n}(\%)\end{array}$ & $\begin{array}{l}\text { Moderate } \\
\text { Preterm, } \\
\mathrm{n}(\%)\end{array}$ & $\begin{array}{l}\text { Early Term, } \\
\mathrm{n}(\%)\end{array}$ & $\begin{array}{l}\text { Full Term, } \\
\mathrm{n}(\%)\end{array}$ & $\begin{array}{l}\text { Late Term, } \\
\text { n (\%) }\end{array}$ & $\begin{array}{l}\text { Post-term, } \\
\text { n (\%) }\end{array}$ & Total \\
\hline $\begin{array}{l}\text { Underweight: } \\
\text { Imputed data }\end{array}$ & $167(0.8)$ & $120(0.6)$ & $225(1.1)$ & $1463(6.9)$ & 4110 (19.5) & $10626(50.4)$ & $3478(16.5)$ & $1028(4.9)$ & 21097 \\
\hline $\begin{array}{l}\text { Underweight: } \\
\text { Original data }\end{array}$ & $77(0.6)$ & $60(0.5)$ & $127(1.0)$ & $888(6.9)$ & $2583(20.2)$ & $6467(50.6)$ & $1993(15.6)$ & $633(5.0)$ & 12768 \\
\hline $\begin{array}{l}\text { Recommended Weight: } \\
\text { Imputed data }\end{array}$ & $1413(0.6)$ & $923(0.4)$ & $1915(0.8)$ & $13728(5.4)$ & 41989 (16.6) & $129263(51.2)$ & 48635 (19.3) & $15632(6.2)$ & 252575 \\
\hline $\begin{array}{l}\text { Recommended Weight: } \\
\text { Original data }\end{array}$ & $1041(0.5)$ & $978(0.3)$ & $1517(0.7)$ & $11451(5.3)$ & $35572(16.3)$ & $112072(51.4)$ & 42364 (19.4) & $13958(6.4)$ & 217975 \\
\hline $\begin{array}{l}\text { Overweight: } \\
\text { Imputed data }\end{array}$ & $848(0.6)$ & $546(0.4)$ & $995(0.8)$ & $6892(5.3)$ & $21802(16.7)$ & $64506(49.4)$ & $26431(20.3)$ & $9044(6.9)$ & 130518 \\
\hline $\begin{array}{l}\text { Overweight: } \\
\text { Original data }\end{array}$ & $563(0.5)$ & $355(0.4)$ & $671(0.7)$ & $5050(4.9)$ & $16636(16.2)$ & $50721(49.4)$ & $21335(20.8)$ & 7701 (7.5) & 102677 \\
\hline $\begin{array}{l}\text { Obese class I: } \\
\text { Imputed data }\end{array}$ & $346(0.7)$ & $212(0.4)$ & $473(0.9)$ & $2861(5.6)$ & 9134 (17.9) & $24243(47.6)$ & $10256(20.1)$ & $3654(7.2)$ & 50967 \\
\hline $\begin{array}{l}\text { Obese class I: } \\
\text { Original data }\end{array}$ & $228(0.6)$ & $136(0.4)$ & $336(0.9)$ & $2077(5.4)$ & $6855(17.8)$ & $18081(46.8)$ & $7991(20.7)$ & 3040 (7.9) & 38608 \\
\hline $\begin{array}{l}\text { Obese class II: } \\
\text { Imputed data }\end{array}$ & $123(0.8)$ & $84(0.5)$ & $158(1)$ & $927(5.8)$ & 3071 (19.1) & 7331 (45.6) & 3145 (19.6) & $1306(8.1)$ & 16061 \\
\hline $\begin{array}{l}\text { Obese class II: } \\
\text { Original data }\end{array}$ & $103(0.7)$ & $71(0.5)$ & $132(0.9)$ & $784(5.6)$ & 2684 (19.1) & $6365(45.2)$ & $2793(19.8)$ & $1216(8.6)$ & 14077 \\
\hline $\begin{array}{l}\text { Obese class IIIa: } \\
\text { Imputed data }\end{array}$ & $50(0.8)$ & $37(0.6)$ & $44(0.7)$ & $334(5.5)$ & $1227(20.3)$ & $2688(44.4)$ & 1202 (19.9) & $508(8.4)$ & 6053 \\
\hline $\begin{array}{l}\text { Obese class Illa: } \\
\text { Original data }\end{array}$ & $49(0.8)$ & $35(0.6)$ & $44(0.7)$ & $328(5.5)$ & $1202(20.3)$ & $2616(44.2)$ & 1177 (19.9) & $502(8.5)$ & 5918 \\
\hline $\begin{array}{l}\text { Obese class IIIb: } \\
\text { Imputed data }\end{array}$ & $7(1.4)$ & $5(1.0)$ & $5(1.0)$ & $48(9.5)$ & $114(22.6)$ & $191(37.9)$ & 89 (17.7) & $50(9.9)$ & 504 \\
\hline $\begin{array}{l}\text { Obese class IIIb: } \\
\text { Original data }\end{array}$ & $7(1.4)$ & $5(1.0)$ & $5(1.0)$ & $48(9.5)$ & $116(22.9)$ & $191(37.7)$ & $90(17.8)$ & $50(9.9)$ & 507 \\
\hline Total: Imputed data & 2954 & 1927 & 3815 & 26254 & 81448 & 238847 & 93237 & 31222 & $477777^{\#}$ \\
\hline Total: Original data & 2068 & 1640 & 2832 & 20626 & 65648 & 196513 & 77743 & 27100 & $392530^{\#}$ \\
\hline
\end{tabular}

\# 2087 (0.4\%) with missing gestational age were excluded. Total including extreme preterm births 20-27 weeks gestation. NOTE: Frequencies for the imputed data were estimated from the imputed percentages, and therefore may not sum exactly due to rounding errors

\section{Post-term birth}

The absolute risk of post-term birth increased monotonically as BMI category increased $(4.9,6.2,6.9,7.2,8.1$, 8.4, and $9.9 \%$ for underweight, recommended, overweight and obese class: I, II, IIIa and IIIb respectively, Table 2). Compared to women in the recommended weight category, the AORs were significantly increased for all obesity classes with the effect sizes increasing almost linearly with increasing maternal BMI category, although there was a steeper increase in effect size for women with class IIIb obesity $(2.03$, 95\% CI 1.48-2.77, Table 3). There was also a significantly increased AOR for maternal overweight and significantly reduced AOR for maternal underweight (Table 3). When considering all obese classes combined, the AOR again showed a similar pattern to the analyses of preterm birth. The AOR was 1.34 (95\% CI 1.30-1.39, Table 4) which falls in between obesity class I and II (AOR 1.23 (95\% CI 1.171.28) and AOR 1.44 (95\% CI 1.35-1.53) respectively, Table 3).

\section{Term birth}

There was a J-shaped association between maternal BMI and absolute risk of early term birth (Table 2). The pattern of AORs mirrored that observed in the preterm birth categories with a significantly increased association for all obesity classes, most notably class IIIb (1.74, 95\%
CI 1.38-2.20) but with generally smaller effect sizes (Table 3). There was also a significantly increased AOR for maternal underweight and borderline significance for overweight categories. The odds of late term birth increased monotonically with increasing maternal BMI, mirroring the association observed between post-term birth and maternal BMI, with a significantly reduced AOR for maternal underweight and linear increase in AORs for maternal overweight through to obesity class IIIb (Table 3).

\section{Discussion}

This study aimed to determine the associations between maternal obesity classes and categories of gestational age at birth. To the best of our knowledge, this was the first study to explore the association between categories of gestational age at birth; in particular extreme preterm birth, and maternal extreme obesity. With the exception of very preterm birth, the results showed a significantly increased association with all obesity classes and gestational age categories. There was a consistent J-shaped association for all preterm and early term birth categories, showing an increased risk for both maternal underweight and obesity, most notably for maternal obesity class IIIb. There was also a linear association with postterm and late term birth categories with a significantly reduced association for maternal underweight, and 
Table 3 Unadjusted and adjusted odd ratios representing the associations between gestational age at delivery and maternal BMI categories

\begin{tabular}{|c|c|c|c|c|c|}
\hline $\begin{array}{l}\text { Gestational age at } \\
\text { delivery }{ }^{\#}\end{array}$ & BMI category & $\begin{array}{l}\text { Unadjusted } \\
\text { OR versus full } \\
\text { term } \\
\text { (imputed data) } \\
(95 \% \mathrm{Cl})^{*}\end{array}$ & $\begin{array}{l}\text { Unadjusted } \\
\text { OR versus full } \\
\text { term (original } \\
\text { data) } \\
(95 \% \mathrm{Cl})^{*}\end{array}$ & $\begin{array}{l}\text { Adjusted* } \\
\text { OR versus full } \\
\text { term } \\
\text { (imputed data) } \\
\text { (95\% Cl) }\end{array}$ & $\begin{array}{l}\text { Adjusted* } \\
\text { OR versus full } \\
\text { term (original } \\
\text { data) } \\
(95 \% \mathrm{Cl})\end{array}$ \\
\hline \multirow{6}{*}{$\begin{array}{l}\text { Extreme Preterm } \\
(20-27 \\
\text { weeks })\end{array}$} & Underweight & $1.43(1.13-1.81)$ & $1.28(1.02-1.62)$ & 1.40 (1.1-1.77) & $1.25(0.93-1.67)$ \\
\hline & Overweight & $1.20(1.1-1.32)$ & $1.20(1.08-1.32)$ & $1.16(1.05-1.27)$ & $1.18(1.04-1.34)$ \\
\hline & Obese class I & $1.31(1.12-1.52)$ & $1.36(1.17-1.57)$ & $1.20(1.03-1.4)$ & $1.24(1.03-1.48)$ \\
\hline & Obese class II & $1.54(1.26-1.88)$ & $1.74(1.42-2.13)$ & $1.39(1.13-1.71)$ & $1.48(1.13-1.92)$ \\
\hline & Obese class IIla & $1.70(1.27-2.26)$ & $2.02(1.51-2.70)$ & $1.52(1.14-2.03)$ & $1.62(1.11-2.37)$ \\
\hline & Obese class IIIb & 3.35 (1.57-7.14) & $3.95(1.85-8.41)$ & $2.80(1.31-5.98)$ & $5.33(2.32-12.23)$ \\
\hline \multirow{6}{*}{$\begin{array}{l}\text { Extreme Preterm } \\
\text { (24-27 weeks) }\end{array}$} & Underweight & $1.58(1.19-2.09)$ & $1.53(1.18-2.00)$ & $1.53(1.16-2.03)$ & $1.46(1.09-1.96)$ \\
\hline & Overweight & $1.19(1.06-1.33)$ & $1.16(1.02-1.32)$ & $1.14(1.02-1.28)$ & $1.14(0.99-1.32)$ \\
\hline & Obese class I & $1.23(1.03-1.46)$ & $1.24(1.03-1.50)$ & $1.13(0.95-1.36)$ & $1.14(0.93-1.40)$ \\
\hline & Obese class II & $1.61(1.27-2.04)$ & $1.84(1.44-2.36)$ & $1.47(1.16-1.87)$ & $1.74(1.33-2.28)$ \\
\hline & Obese class IIIa & $1.9(1.36-2.66)$ & $2.21(1.57-3.11)$ & $1.74(1.24-2.45)$ & $2.10(1.45-3.04)$ \\
\hline & Obese class IIIb & $3.67(1.51-8.94)$ & $4.33(1.78-10.54)$ & $3.17(1.3-7.74)$ & $4.61(1.88-11.29)$ \\
\hline \multirow{6}{*}{$\begin{array}{l}\text { Very Preterm } \\
\text { (28-31 weeks) }\end{array}$} & Underweight & $1.43(1.23-1.66)$ & $1.45(1.21-1.74)$ & $1.37(1.18-1.59)$ & $1.53(1.23-1.91)$ \\
\hline & Overweight & $1.04(0.95-1.14)$ & $0.98(0.89-1.07)$ & $1.00(0.91-1.09)$ & $0.94(0.83-1.05)$ \\
\hline & Obese class I & 1.32 (1.17-1.49) & $1.37(1.22-1.54)$ & $1.21(1.07-1.37)$ & $1.23(1.06-1.44)$ \\
\hline & Obese class II & 1.45 (1.21-1.74) & $1.53(1.28-1.83)$ & $1.35(1.12-1.61)$ & $1.41(1.12-1.77)$ \\
\hline & Obese class IIIa & $1.10(0.81-1.5)$ & $1.24(0.92-1.68)$ & $1.04(0.76-1.42)$ & $1.28(0.89-1.84)$ \\
\hline & Obese class IIIb & $1.77(0.73-4.30)$ & $1.93(0.79-4.70)$ & $1.59(0.65-3.88)$ & $1.31(0.32-5.32)$ \\
\hline \multirow{6}{*}{$\begin{array}{l}\text { Moderately Preterm } \\
\text { (32-36 weeks) }\end{array}$} & Underweight & $1.30(1.20-1.40)$ & $1.34(1.20-1.32)$ & $1.28(1.19-1.38)$ & $1.31(1.19-1.43)$ \\
\hline & Overweight & $1.01(0.97-1.04)$ & $0.97(0.94-1.01)$ & $0.99(0.96-1.02)$ & $0.95(0.92-1.00)$ \\
\hline & Obese class I & $1.11(1.06-1.16)$ & $1.12(1.07-1.18)$ & $1.07(1.02-1.12)$ & $1.04(0.98-1.11)$ \\
\hline & Obese class II & $1.19(1.1-1.28)$ & $1.21(1.12-1.30)$ & $1.13(1.05-1.22)$ & $1.13(1.03-1.26)$ \\
\hline & Obese class IIIa & $1.17(1.04-1.32)$ & $1.23(1.09-1.38)$ & $1.11(0.99-1.25)$ & $1.18(1.03-1.25)$ \\
\hline & Obese class IIIb & $2.37(1.72-3.25)$ & $2.46(1.79-3.38)$ & $2.18(1.58-2.99)$ & $2.40(1.56-3.68)$ \\
\hline \multirow{6}{*}{$\begin{array}{l}\text { Early Term } \\
\text { (37-38 weeks) }\end{array}$} & Underweight & $1.19(1.14-1.25)$ & $1.26(1.20-1.32)$ & $1.19(1.14-1.25)$ & $1.28(1.21-1.37)$ \\
\hline & Overweight & $1.04(1.02-1.06)$ & $1.03(1.01-1.06)$ & $1.02(1.00-1.04)$ & $1.00(0.97-1.37)$ \\
\hline & Obese class I & $1.16(1.12-1.2)$ & $1.19(1.56-1.23)$ & $1.12(1.08-1.16)$ & $1.12(1.07-1.16)$ \\
\hline & Obese class II & $1.29(1.23-1.35)$ & $1.33(1.27-1.39)$ & $1.24(1.18-1.3)$ & $1.24(1.17-1.32)$ \\
\hline & Obese class IIla & $1.41(1.31-1.51)$ & $1.45(1.35-1.55)$ & $1.35(1.26-1.45)$ & $1.32(1.21-1.45)$ \\
\hline & Obese class IIIb & $1.84(1.46-2.32)$ & $1.91(1.52-2.41)$ & $1.74(1.38-2.20)$ & $1.97(1.44-2.70)$ \\
\hline \multirow{6}{*}{$\begin{array}{l}\text { Late term } \\
\text { (41 weeks) }\end{array}$} & Underweight & $0.87(0.83-0.91)$ & $0.82(0.77-0.86)$ & $0.86(0.82-0.91)$ & $0.80(0.74-0.85)$ \\
\hline & Overweight & $1.09(1.07-1.11)$ & $1.11(1.18-1.25)$ & $1.09(1.07-1.11)$ & $1.12(1.09-1.15)$ \\
\hline & Obese class I & $1.12(1.09-1.16)$ & $1.17(1.14-1.20)$ & $1.12(1.09-1.15)$ & $1.15(1.11-1.20)$ \\
\hline & Obese class II & $1.14(1.09-1.2)$ & $1.16(1.11-1.28)$ & $1.13(1.07-1.18)$ & $1.12(1.05-1.19)$ \\
\hline & Obese class IIIa & $1.19(1.11-1.28)$ & 1.19 (1.11-1.28) & $1.17(1.09-1.25)$ & $1.15(1.05-1.26)$ \\
\hline & Obese class IIIb & $1.24(0.96-1.59)$ & $1.25(0.97-1.60)$ & $1.18(0.92-1.52)$ & $1.42(1.02-1.97)$ \\
\hline \multirow{6}{*}{$\begin{array}{l}\text { Post-term } \\
\text { ( } \geq 42 \text { weeks) }\end{array}$} & Underweight & $0.80(0.73-0.87)$ & $0.79(0.72-0.85)$ & $0.78(0.72-0.85)$ & $0.78(0.71-0.87)$ \\
\hline & Overweight & $1.16(1.13-1.19)$ & $1.22(1.18-1.26)$ & $1.16(1.13-1.19)$ & $1.22(1.18-1.27)$ \\
\hline & Obese class I & $1.25(1.19-1.3)$ & $1.35(1.29-1.41)$ & $1.23(1.17-1.28)$ & $1.36(1.29-1.43)$ \\
\hline & Obese class II & $1.47(1.38-1.57)$ & $1.53(1.44-1.63)$ & $1.44(1.35-1.53)$ & $1.50(1.39-1.62)$ \\
\hline & Obese class IIla & $1.56(1.42-1.72)$ & $1.54(1.40-1.70)$ & $1.53(1.38-1.68)$ & $1.52(1.35-1.71)$ \\
\hline & Obese class IIIb & $2.16(1.58-2.96)$ & $2.10(1.54-2.87)$ & $2.03(1.48-2.77)$ & $2.45(1.66-3.62)$ \\
\hline
\end{tabular}

\# Full term birth (39-40 weeks) was the reference group for all gestational age comparisons

*Recommended BMI was the reference group for all BMI comparisons

$¥$ Adjusted for BMI category, IMD quintile, ethnicity, employment, maternal age, parity, gestational age at booking and year of deliver 
Table 4 Unadjusted and adjusted odd ratios (estimated using imputed data) representing the associations between gestational age at delivery and combined maternal obesity classes (BMI $\geq 30 \mathrm{kgm} 2)^{*}$

\begin{tabular}{|c|c|c|c|c|}
\hline Gestational age category\# & $\begin{array}{l}\text { Unadjusted OR } \\
(95 \% \mathrm{CI}) \\
\text { (imputed data) }\end{array}$ & $\begin{array}{l}\text { Unadjusted OR } \\
(95 \% \mathrm{Cl}) \\
\text { (original data) }\end{array}$ & $\begin{array}{l}\text { Adjusted OR } ¥ \\
(95 \% \mathrm{Cl}) \\
\text { (imputed data) }\end{array}$ & $\begin{array}{l}\text { Adjusted OR } ¥ \\
\text { (95\% CI) } \\
\text { (original data) }\end{array}$ \\
\hline Extreme preterm & $1.41(1.24-1.59)$ & $1.53(1.36-1.72)$ & $1.33(1.17-1.52)$ & $1.35(1.16-1.57)$ \\
\hline Very preterm & $1.32(1.20-1.47)$ & $1.40(1.27-1.55)$ & $1.24(1.11-1.37)$ & $1.28(1.12-1.45)$ \\
\hline Moderately preterm & $1.14(1.10-1.19)$ & $1.16(1.11-1.21)$ & $1.10(1.06-1.14)$ & $1.08(1.03-1.14)$ \\
\hline Early term & $1.21(1.18-1.25)$ & 1.25 (1.22-1.29) & $1.17(1.14-1.20)$ & $1.17(1.13-1.21)$ \\
\hline Late term & $1.14(1.11-1.16)$ & $1.17(1.14-1.20)$ & $1.15(1.12-1.18)$ & $1.14(1.11-1.18)$ \\
\hline Post term & $1.32(1.28-1.37)$ & $1.41(1.36-1.46)$ & $1.34(1.30-1.39)$ & $1.40(1.34-1.47)$ \\
\hline
\end{tabular}

\# Full term birth was the reference group for all gestational age comparisons

*Recommended BMI was the reference group for all BMI comparisons

$¥$ Adjusted for BMI category, IMD quintile, ethnicity, employment, maternal age, parity, gestational age at booking and year of delivery

increasing association with obesity classes; again most notably with obesity class IIIb.

The associations identified in this research add evidence to the argument that combining subclasses of maternal obesity does not accurately represent the risks for many women with obesity. Doing so resulted in an overestimation of risk of preterm and post-term birth for women with class I obesity, and an underestimation of risk for women in all other obesity classes. This is especially the case for women with class IIIb obesity. Two previous studies reporting on class IIIb obesity also showed increased risks including pre-eclampsia, gestational diabetes, admission to intensive care and caesarean section, large for gestational age and a 5-min Apgar score $<7[14,15]$. Risks were increased compared with all other BMI categories, including women with obesity ( $>30$ to $<50 \mathrm{~kg} / \mathrm{m}^{2}$ ). We also found that women with class IIIb obesity were at the greatest risks of both preterm and post-term birth. In particular, this population had almost 3-fold increased odds of extreme preterm birth (20-27 weeks gestation) and more than 3-fold increase when restricting to the gestation for viability (2427 weeks). There are significant inequalities associated with class IIIb obesity, especially relating to socioeconomic status. UK data shows that women with class IIIb obesity have almost five-times increased odds of living in areas of highest deprivation than women with a recommended BMI, following adjustment for additional socio-demographic confounders [13]. Therefore, our findings reflect a double burden of inequality for mothers who face the highest levels of deprivation and the greatest risk of adverse preterm and post-term outcomes, and subsequent inequality for lifelong health of their offspring. For all preterm infants, costs associated with the provision of neonatal intensive care are increased [32]. In addition, the low chance of survival for infants born below the limit of viability [26] raises a number of difficult decisions for both parents and health professionals in relation to resuscitation [33]. Although obesity alone may not be an indication for induction of labour, the association between maternal obesity and post-term birth observed by both this study and other published research suggests an increase in risk of the requirement for either induction of labour or increased antenatal monitoring.

Our study was not able to distinguish between spontaneous and medically indicated preterm births. This is important given that women with obesity are more likely to develop comorbidities such as gestational diabetes or pre-eclampsia, which can necessitate early delivery [34]. A study in the US investigated the risk of spontaneous and indicated preterm birth for women with class I obesity, and combined classes II/III [35]. The authors from the US study identified significantly increased relative risks (RR) for both classes (obesity class I, and combined classes II/III) and both spontaneous and indicated extreme preterm birth (defined as 23-27 weeks) compared to term birth (defined as $\geq 37$ weeks). They reported a significantly increased RR for both obesity classes and indicated moderate/late preterm birth (3236 weeks) and also for class II/III obesity and indicated very preterm birth (28-31 weeks). However, they found no significant increased risk for either of the obesity classes and spontaneous very or moderate/late preterm birth, or for indicated very preterm birth for class II/III obesity. Our study adds to this evidence by investigating both the sub-classes of obesity and subcategories of term birth.

\section{Strengths and limitations}

This study used national data for England and included a large sample of 479,864 live births from eight regions, which had $99.6 \%$ complete gestational age data. The large sample size meant we had the power to investigate important sub-classes of both maternal obesity including extreme obesity, and gestational age including extreme preterm birth. Although we did not do any power calculation for the analysis, previous studies investigating 
maternal extreme obesity used samples of 665 and 370 women with extreme obesity. Knight et al found that in a sample of 764,387 women in the UK, 665 had extreme obesity (estimated a prevalence of 8.7 per cases of extreme obesity per 10,000 pregnant women) [14]. Sullivan et al identified 370 women with extreme obesity in Australia (from approximately 171,289 women giving birth) [36]. With these sample sizes, both studies were able to consider the association between pregnancy outcomes and extreme obesity. Based on the prevalence estimate for a UK population by Knight et al., [14] in our sample we would expect 418 cases of extreme obesity (the actual number of cases in our study exceeded this: $n=508$ women).

To our knowledge, this is the first study of this kind. We were able to show that the risk of preterm and postterm birth is not uniform in mothers with a BMI $\geq$ $30 \mathrm{kgm}^{2}$, but increases with increasing obesity classes. Crucially, we were able to analyse the association between maternal obesity class IIIb and extreme preterm birth; this is a novel finding and important to maternal services given the rapid increase in the prevalence of class III obesity over time [13] and the large effect size observed. The sample size was also sufficient to apply the recommended subcategories of term birth, an underresearch area in the context of maternal obesity, and showed a varied association. Our results showed that although that there may be little clinical significance in subdividing term birth for women with overweight and obesity, this may be a useful approach for women with an underweight BMI as there was a higher proportion of women in this BMI group delivering early term.

We had access to multiple socio-demographic variables enabling us to control for important confounding factors and reasonably impute missing values under the missing at random assumption. The use of multiple imputation with chained equations, as opposed to complete case analysis, meant we were able to reduce bias in our estimates due to missingness, while also maximising our sample size. However, the dataset did not contain information on other variables, which may be important confounders. For example, we may have introduced unobserved confounding because we were not able to adjust for smoking, which is associated with preterm birth [37] and maternal BMI [38]. Finally, the aim of this study was to investigate both obesity sub-classes and the subcategories of gestational age, resulting in multiple testing of outcomes. Due to random variation, we would expect one in twenty comparisons to be statistically significant at the $5 \%$ level and multiple comparisons increase the likelihood that a statistically significant result may be observed [39]. Caution should be taken when interpreting the $p$-values; although the effect sizes show evidence that the odds of pre- and post-term birth increase across maternal BMI categories. Due to the large sample size it is also important to keep in mind that small absolute differences may be statistically significantly for example although women with class IIIb obesity have approximately a 3 -fold increase in risk for extreme preterm birth, this outcome is rare, and only $1.4 \%$ of all women have an extreme preterm birth.

\section{Conclusion}

There is a J-shaped association between maternal BMI and extreme preterm, very preterm, moderate preterm and early term birth, and a linear association with late term and post-term birth. While few studies have researched class IIIb obesity, we found that this population had the greatest odds of preterm and post-term birth, particularly for extreme preterm birth, which results in the greatest risks for offspring survival and lifelong health. Given the strong association with class IIIb obesity and deprivation, these women and their children are at particular risk of health inequalities and should be a priority for future research, public health and clinical guidelines.

We have added evidence to the need for pregnancyrelated research and practice to consider all obesity classes separately; obesity is a heterogeneous population as not all obesity classes have same level of risk or care requirements. Additionally, we have shown that early, full and late term births have very different associations with maternal BMI, where early term reflects the patterns observed among preterm birth, and late term reflects the patterns observed with post-term birth. Term birth subcategories should therefore be considered separately in line with the 2013 guideline recommendations when estimating risk of gestational age as an outcome, and also based on evidence that new-born outcomes are not uniform after 37 weeks [19].

\section{Abbreviations \\ AOR: Adjusted odds ratio; BMI: Body Mass Index; Cl: Confidence interval; IMD: Index of multiple deprivation; NHS: National Health Service; OR: Odds ratio; UK: United Kingdom; USA: United States of America; WHO: World Health Organisation}

\section{Acknowledgements}

We would like to acknowledge the staff at the NHS maternity services that provided data for the initial dataset used in this secondary analysis.

\section{Authors' contributions}

All authors were involved in the conception and planning of this research. $\mathrm{NH}$ and JR acquired the original dataset. $\mathrm{NH}, \mathrm{ES}$ and $\mathrm{KB}$ cleaned and coded the data. KB and ES analysed the data. All authors contributed to writing this manuscript. All authors have approved and read the manuscript.

\section{Funding}

This research was funded by a Medical Research Council and Newcastle University Faculty of Medical Sciences Doctoral Training award for ES MR/ K501396/1. The funding body did not contribute to the design of the study, the collection, analysis and interpretation of data or the writing of the manuscript. 


\section{Availability of data and materials}

Data used in this study are not currently publicly available. Requests for access to the dataset should be made to the corresponding author, and will require all necessary ethical approvals and data sharing agreements to be in place.

\section{Ethics approval and consent to participate}

Favourable ethical approval for the secondary analysis of this anonymised dataset was received on the 7th April 2014 from the Proportionate Review Sub-committee of the NHS Health Research Authority National Research Ethics Service (NRES) Committee North West, Preston (REC 14/NW/0249). As this was a retrospective study of routine anonymised medical records, participant

informed consent was waived. Approvals were granted by the Research and Development (R\&D) departments for all NHS Trusts to provide the routine anonymised data in the generation of the original dataset that was used in this secondary analysis.

\section{Consent for publication}

Not applicable.

\section{Competing interests}

Nicola Heslehurst is a member of the editorial board (Associate Editor). The other authors declare that they have no competing interests.

\section{Received: 1 April 2019 Accepted: 7 November 2019}

\section{Published online: 21 November 2019}

\section{References}

1. Liu L, Oza S, Hogan D, Chu Y, Perin J, Zhu J, et al. Global, regional, and national causes of under-5 mortality in 2000-15: an updated systematic analysis with implications for the sustainable development goals. Lancet (London, England). 2016;388(10063):3027-35.

2. Blencowe H, Cousens S, Oestergaard MZ, Chou D, Moller A-B, Narwal R, et al. National, regional, and worldwide estimates of preterm birth rates in the year 2010 with time trends since 1990 for selected countries: a systematic analysis and implications. Lancet. 2012;379(9832):2162-72.

3. Flenady V, Koopmans L, Middleton P, Frøen JF, Smith GC, Gibbons K, et al. Major risk factors for stillbirth in high-income countries: a systematic review and meta-analysis. Lancet. 2011;377(9774):1331-40.

4. Shea KM, Wilcox AJ, Little RE. Postterm delivery: a challenge for epidemiologic research. Epidimiology. 1998;9(2):199-204.

5. Caughey AB, Musci TJ. Complications of term pregnancies beyond 37 weeks of gestation. Obstet Gynecol. 2004;103(1):57-62.

6. Siozos C, Stanley KP. Prolonged pregnancy. Curr Obstet Gynaecol. 2005; 15(2):73-9.

7. Arrowsmith S, Wray S, Quenby S. Maternal obesity and labour complications following induction of labour in prolonged pregnancy. BJOG. 2011;118(5): 578-88.

8. The American College of Obstetricians and Gynecologists. Practice bulletin 55. Management of Post-term pregnancy. Obstet Gynecol. 2004; 104(3):639-46

9. Caughey $A B$, Stotland NE, Washington $A E$, Escobar GJ. Who is at risk for prolonged and postterm pregnancy? Am J Obstet Gynecol. 2009;200(6): 683.e1-5.

10. National Maternity \& Perinatal Audit. Clinical report- revised version; 2017.

11. Ogden CL, Carroll MD, Kit BK, Flegal KM. Prevalence of childhood and adult obesity in the United States, 2011-2012. Jama. 2014;311(8):806-14.

12. Torloni MR, Betran AP, Horta BL, Nakamura MU, Atallah AN, Moron AF, et al. Prepregnancy BMI and the risk of gestational diabetes: a systematic review of the literature with meta-analysis. Obes Rev. 2009;10(2):194-203.

13. Heslehurst N, Rankin J, Wilkinson JR, Summerbell CD. A nationally representative study of maternal obesity in England, UK: trends in incidence and demographic inequalities in 619323 births, 1989-2007. Int J Obes (Lond). 2010;34(3):420-8.

14. Knight M, Kurinczuk JJ, Spark P, Brocklehurst P. Extreme obesity in pregnancy in the United Kingdom. Obstet Gynecol. 2010;115(5):989-97.

15. Alanis MC, Goodnight WH, Hill EG, Robinson CJ, Villers MS, Johnson DD. Maternal super-obesity (body mass index $\geq 50$ ) and adverse pregnancy outcomes. Acta Obstet Gynecol Scand. 2010;89(7):924-30.
16. Torloni MR, Betran AP, Daher S, Widmer M, Dolan SM, Menon R, et al. Maternal BMl and preterm birth: a systematic review of the literature with meta-analysis. J Matern-Fetal Neonatal Med. 2009;22(11):957-70.

17. McDonald SD, Han Z, Mulla S, Beyene J, Knowledge SG. Overweight and obesity in mothers and risk of preterm birth and low birth weight infants: systematic review and meta-analyses. Br Med J. 2010;341:C3428.

18. Heslehurst N, Vieira R, Hayes L, Crowe L, Jones D, Robalino S, et al. Maternal body mass index and post-term birth: a systematic review and metaanalysis. Obes Rev. 2017;18(3):293-308.

19. Spong CY. Defining "term" pregnancy: recommendations from the defining "term" pregnancy workgroup. Jama. 2013;309(23):2445-6.

20. Barber $C$, Rankin J, Heslehurst N. Maternal body mass index and access to antenatal care: a retrospective analysis of 619,502 births in England. BMC Pregnancy Childbirth. 2017;17(1):290.

21. Heslehurst N, Sattar N, Rajasingam D, Wilkinson J, Summerbell CD, Rankin J. Existing maternal obesity guidelines may increase inequalities between ethnic groups: a national epidemiological study of 502,474 births in England. BMC Pregnancy Childbirth. 2012;12:156.

22. Noble M, Wright G, Smith G, Dibben C. Measuring multiple deprivation at the small-area level. Environ Plan A. 2006;38(1):169.

23. Dept for Communities \& Local Government. English indices of deprivation 2010; 2010. [Available from: https://www.gov.uk/government/uploads/ system/uploads/attachment_data/file/6222/1871538.pdf

24. American College of Obstetrics and Gynecologists. Definition of term birth. Comittee Opinion No. 579. Obstet Gynecol. 2013;(122):1139-40.

25. Office for National Statistics. Gestation-specific infant mortality in England and Wales, 2010; 2012

26. Wood NS, Costeloe K, Gibson AT, Hennessy EM, Marlow N, Wilkinson AR. The EPICure study: associations and antecedents of neurological and developmental disability at 30 months of age following extremely preterm birth. Arch Dis Child Fetal Neonatal Ed. 2005;90(2):F134-F40.

27. Crane JMG, Murphy P, Burrage L, Hutchens D. Maternal and perinatal outcomes of extreme obesity in pregnancy. J Obstet Gynaecol Can. 2013; 35(7):606-11.

28. Henry CJK. Body mass index and the limits of human survival. Eur J Clin Nutr. 1990;44(4):329-35.

29. Centre for Maternal and Child Enquiries (CMACE). Maternal obesity in the UK: findings from a national project. London: CMACE; 2010.

30. Peacock J, Peacock P. Chapter 12 Analysisng multiple variables per subject. In: Oxford handbook of medical statistics. Oxford: Oxford University Press; 2011. p. 393-445.

31. White IR, Royston P, Wood AM. Multiple imputation using chained equations: issues and guidance for practice. Stat Med. 2011;30(4):377-99.

32. Saigal S, Doyle LW. An overview of mortality and sequelae of preterm birth from infancy to adulthood. Lancet. 2008;371(9608):261-9.

33. Macfarlane PI, Wood S, Bennett J. Non-viable delivery at 20-23 weeks gestation: observations and signs of life after birth. Arch Dis Child Fetal Neonatal Ed. 2003;88(3):F199-202.

34. Cnattingius S, Villamor E, Johansson S, Bonamy AKE, Persson M, Wikstrom AK, et al. Maternal obesity and risk of preterm delivery. JAMA. 2013;309(22):2362-70.

35. Kim S, Mendola P, Zhu Y, Hwang B, Grantz K. Spontaneous and indicated preterm delivery risk is increased among overweight and obese women without prepregnancy chronic disease. BJOG. 2017;124(11):1708-16.

36. Sullivan EA, Dickinson JE, Vaughan GA, Peek MJ, Ellwood D, Homer CSE, et al. Maternal super-obesity and perinatal outcomes in Australia: a national population-based cohort study. BMC Pregnancy Childbirth. 2015;15(1):322.

37. Cnattingius $\mathrm{S}$. The epidemiology of smoking during pregnancy: smoking prevalence, maternal characteristics, and pregnancy outcomes. Nicotine Tob Res. 2004;6(Suppl 2):S125-S40.

38. Tan AKG, Yen ST, Feisul MI. The association between smoking and body mass index: results from a national sample of Malaysian adults. J Public Health. 2013;21(5):403-12.

39. Kirkwood BR, Sterne AC. Common pitfalls in analysis and interpretation. Essential medical statistics. Oxford: Blackwell Publishing Ltd; 2003. p. 467.

\section{Publisher's Note}

Springer Nature remains neutral with regard to jurisdictional claims in published maps and institutional affiliations. 